\title{
CARACTERIZACIÓN DEL CONSUMO DE ALCOHOL EN ESTUDIANTES DE UNA INSTITUCIÓN PRIVADA EN UNA REGIÓN DE PERÚ
}

\author{
Characterization of alcohol consumption in students of a private institution in a region of Perú
}

\author{
Héctor William Carlos Cruces ${ }^{1, a, e}$, Belinda Marleni Navarro Guerra ${ }^{1, f}$, Nidia Magali Palacios \\ Fuentes. ${ }^{2 c, d}$ ThaliaJesalem Carlos Campos ${ }^{2 b}$
}

\begin{abstract}
${ }^{1}$ Docente de la Facultad de Ciencias Económicas y Negocios Internacionales, Universidad Nacional San Luis Gonzaga. Ica, Perú ${ }^{2}$ Hospital Augusto Hernández Mendoza Es Salud - Ica, Perú

${ }^{a}$ Economista, ${ }^{b}$ Licenciada en Enfermería, ${ }^{\circ}$ Obstetra, ${ }^{d} \mathrm{Mg}$ en Salud Pública, ${ }^{e}$ Doctor en Educación, Doctor en Ciencias Administrativas
\end{abstract}

\section{RESUMEN}

El estudio tuvo como Objetivo: Determinar los factores socio familiares y su influencia en el consumo de alcohol de los adolescentes en la Institución Educativa Particular Jesús Maestro-Ica Perú Material y Métodos: El diseño del estudio fue no experimental, cuantitativo, explicativo, aplicada de corte transversal. Muestra constituida por 174 estudiantes. Utilizando un cuestionario para estudiantes adolescentes Resultados: En relación a estructura, violencia, relación , y antecedentes de consumo familiar, un $48 \%$ es desfavorable, en cuanto a los factores interpersonales fue desfavorable en un $60 \%$, un $73 \%$ consume alcohol antes de los 15 años, por curiosidad $94 \%$, el consumo actual es por presión de los amigos $97 \%$, fácil acceso al alcohol $67 \%$, frecuencia de consumo de dos veces por semana $41 \%$, en el cual un 59\% consume en lugares públicos Conclusiones: El estudio hace hincapié en que un $48 \%$ de los factores socio familiares son desfavorables, donde un $55,7 \%$ de los estudiantes tienen un alto consumo de alcohol, un $23,7 \%$ un consumo medio y solo un $20,6 \%$ tiene un consumo bajo de alcohol.

Palabras clave: Consumo de alcohol, Factores socio-familiares, conducta de riesgo, sistema educativo

\section{SUMMARY}

The objective of the study was: To determine the socio-family factors and their influence on adolescent alcohol consumption in the Private Educational Institution Jesús Maestro-Ica Peru Material and Methods: The design of the study was non-experimental, quantitative, explanatory, applied cross-cutting. Sample consisting of 174 students. Using a questionnaire for teenage students Results: In relation to structure, violence, relationship, and family consumption history, $48 \%$ is unfavorable, as far as interpersonal factors were unfavorable by $60 \%, 73 \%$ consume alcohol before age 15 , out of curiosity $94 \%$, current consumption is by pressure of friends $97 \%$, easy access to alcohol $67 \%$, frequency of consumption twice a week $41 \%$, in which $59 \%$ consume in public lugers Conclusions: The study emphasizes that $48 \%$ of sociofamily factors are unfavorable, where $55.7 \%$ ofstudents have high alcohol consumption, $23.7 \%$ average consumption and only $20.6 \%$ have low alcohol consumption

Keywords: Alcohol consumption, Sociofamily factors, risky behavior, education system

\section{INTRODUCCIÓN}

Desde la antigüedad, en todas las culturas, el consumo de bebidas alcohólicas siempre ha existido a lo largo de la historia de la humanidad. Desde la segunda mitad del siglo
Veinte, existen algunos factores sociales que inciden en la prevalencia del alcoholismo, que es un problema general, como un buen ambiente social, el consumo y adquisición de bebidas alcohólicas, evitando problemas 
personales y tradiciones socioculturales que propician el consumo. El uso nocivo de alcohol constituye un problema social ya que tiene un impacto negativo en las vidas de un segmento considerable de la población, el mismo para su estudio y comprensión podemos analizarlo a través de dos funciones las que se manifiestan y las latentes. Como lo hace notar la Organización Mundial de la Salud (OMS), más de 3 millones de personas murieron por el uso nocivo de alcohol en 2016, lo que representa una de cada 20 muertes, más de las tres cuartas partes de las muertes fueron hombres, en general, el consumo nocivo de alcohol representa más del $5 \%$ de la carga mundial de morbilidad, se estima que en el mundo hay 237 millones de hombres y 46 millones de mujeres que padecen trastornos por consumo de alcohol.(1) La mayor prevalencia entre hombres y mujeres se registra en la Región de Europa (14,8\% y $3,5 \%$ ) y la Región de las Américas (11,5\% y $5,1 \%)$. Los trastornos por consumo de alcohol son más frecuentes en los países de ingresos altos (1). De todas las muertes atribuibles al alcohol, el $28 \%$ se debieron a lesiones, como las causadas por accidentes de tránsito, autolesiones y violencia interpersonal; el $21 \%$ a trastornos digestivos; el $19 \%$ a enfermedades cardiovasculares, y el resto a enfermedades infecciosas, cánceres, trastornos mentales y otras afecciones (1), el consumo de sustancias psicoactivas se ha incrementado enormemente, lo que constituye un fenómeno público real y un grave problema de salud pública. Tanto mujeres como hombres presentan un aumento al consumo de alcohol, a medida que avanzan en los grados de estudio, ambos sexos comparten prevalencias anuales significativas de consumo de alcohol en el grupo de edad de 11 a 13 años y primer año de secundaria; Con altas probabilidades de consumir otras sustancias dañinas para la salud (2). En el Perú, el alcohol es la droga más consumida entre los adolescentes, desde el establecimiento de mediciones nacionales estandarizadas y comparables en la población escolar de nivel secundario, el consumo de alcohol ha registrado las mayores prevalencias, se ha estimado que el $29,5 \%$ de los escolares de nivel secundario (alrededor de 438000 adolescentes) refiere haber usado alcohol alguna vez en la vida y el $17,5 \%$ (aproximadamente 259000 estudiantes) reporta haber usado alcohol en los últimos doce meses. (3). Los problemas con el alcohol se deben a múltiples causas; la literatura al respecto coincide en ciertos macro factores que son motivadores del consumo entre los adolescentes: hedonismo, búsquedas personales o surgidas del grupo de pares, exploración de experiencias diversas, redefinición de la identidad e integración grupal a través de rituales de ocio (4). Un modelo más complejo, pero a la vez más holístico, plantea diversos niveles determinantes del consumo de alcohol (4): lo intraindividual (genética, predisposiciones individuales, factores motivacionales), los factores micro sociales (refuerzo social, redes, modelos negativos del grupo de iguales o familia, presión social), lo individual (rasgos de personalidad), los factores de carácter contextual (modelado sociocultural, inercia social, disponibilidad de alcohol, publicidad), y la acción de los mecanismos de control (sociales, políticos o fácticos), significa que los factores de riesgos pueden presentarse en la escuela, entorno familiar, amigos, o en la misma personalidad del sujeto, donde la existencia de factores protectores reducirá la posibilidad de beber o abuso de bebidas alcohólicas. Las relaciones de pares son más críticas en la adolescencia, que en cualquier otro momento del desarrollo (4). El consumo de alcohol se presenta cada vez a edades más tempranas y al parecer aceptado por la familia, lo cual potencializa el daño y la conducta adictiva (5).

Así mismo la comparación de consumo de alcohol de adolescentes de dos instituciones educativas, una pública y otra privada revela que los adolescente han iniciado su consumo de alcohol en la institución pública a la edad de 10 años 54\%, teniendo como razón de consumo inicial la curiosidad declara un $71 \%$, 
por otro lado señalan que su consumo actual es con los amigos $42 \%$, a diferencia de la institución privada inicio su consumo de alcohol a los 9 años lo que representa un 46\%, así mismo declaran en mayor proporción que iniciaron el consumo de bebidas alcohólicas por la invitación de un amigo un 69\%, también manifiestan que la razón del consumo actual de bebidas alcohólicas lo hace con amigos representando un $62 \%$.(6). Vinculado a un conjunto de consecuencias negativos en el ámbito educativo, tales como la falta de motivación en la escuela, el fracaso escolar o el abandono de los estudios, tiene un impacto en los esfuerzos que lleva a cabo el sector de la educación para garantizar una educación de calidad inclusiva y equitativa para todos, así como en la consecución de la Agenda mundial 2030 para el desarrollo sostenible. El sector de la educación tiene, por consiguiente, la responsabilidad fundamental de proteger a los niños y a los jóvenes del consumo de sustancias psicoactivas.; el problema surge cuando la institución no actúa de manera preventiva ante el consumo de alcohol y se vuelven indolentes ante este problema; se necesita docentes entrenados e instituciones involucradas en la problemática. Existen muchos factores que contribuyen a los problemas relacionados con el alcohol, los impactos sociales y ambientales, como las costumbres y actitudes que abogan por un alto consumo (7).

Se justifica el estudio toda vez que el consumo nocivo de alcohol es uno de los principales factores de riesgo de los trastornos neuro psiquiátricos y otras enfermedades no transmisibles, como las cardiovasculares, la cirrosis hepática y diversos cánceres, también el uso nocivo del alcohol está relacionado con varias enfermedades infecciosas, como el $\mathrm{VIH} /$ sida, la Tuberculosis y la neumonía. Las consecuencias del consumo de alcohol por los estudiantes adolescentes se acrecientan, es imperante determinar los efectos que traerá para el adolescente el consumo desmedido de alcohol y sobre todo el rol del educador y la familia en la, prevención del consumo de alcohol, siendo de importancia el abordaje de este tema por la implicancia en la salud pública, la deserción y bajo rendimiento de los estudiantes. Lo expuesto motivó a investigar, señalándose como objetivo determinar los factores socios familiares y su influencia en el consumo de alcohol de los adolescentes en la Institución Educativa Particular Jesús MaestroIca-Perú.

\section{MATERIAL Y MÉTODOS}

La población de estudio fue conformada por 1108 estudiantes adolescentes asistentes a la institución Educativa particular Jesús Maestro, el tamaño de la muestra se obtuvo con fórmula estadística, en total 174 estudiantes. Se utilizó la técnica de la encuesta y como herramienta de recolección de datos el cuestionario escrito y auto administrado, estructurado, que se aplicó a los adolescentes de dicha institución, cuyo formulario consta de datos generales y el cuerpo central en la que se incluyen los 20 ítems referentes a las variables y de acuerdo a los objetivos de estudio, instrumento validado por juicio de expertos, investigación de tipo aplicada, porque va a solucionar problemas facticos como el consumo de alcohol y los factores familiares en estudiantes del colegio privado Jesús Maestro (8), Se utilizaron los métodos siguientes: deductivo que determinó el problema, analítico, que analizó los resultados, así mismo el sintético, que formuló conclusiones y recomendaciones, también el bibliográfico, que extrajo la información en textos e Internet, y el descriptivo, que describió cada paso de la investigación, estudio de nivel explicativo; diseño no experimental, de corte transeccional, correlacional.

\section{RESULTADOS}

Se presentan los resultados de acuerdo al cuestionario aplicado. En cada sección se presentan los resultados del análisis descriptivo y de la inferencia estadística, exponiendo la información según detalle. 
En el gráfico 1. Se puede observar que los factores familiares fueron favorables alcanzando $\quad 66 \%(115 \quad$ estudiantes) destacándose que 80\%(139 estudiantes ) tiene familia nuclear, con una media aritmética de 033, error típico de 0,23, mediana de 0,12, desviación estándar de 0,40 y varianza de la muestra de 0,16, un 82\%(142 estudiantes) califican de buena su relación familiar, con una media aritmética de 0,66, error típico de 0,25, mediana de 0,82, desviación estándar de 0,43 y varianza de la muestra de 0,19 , así mismo un $79 \%$ (137 estudiantes) manifiesta que no existe violencia familiar, con una media aritmética de 0,5,con un error típico de 0,29, mediana de 0,5 , desviación estándar de 0,41 y una varianza de la muestra de 0,16 y el $86 \%(150$ estudiantes ) menciona que no existe antecedente familiar respecto a consumo de alcohol, con una media aritmética de 0,5 ,con un error típico de 0,36, mediana de 0,5 , desviación estándar de 0,5 y una varianza de la muestra de 0,25.

En el gráfico 2. En relación a los factores interpersonales de los estudiantes es desfavorable en un $60 \%$, donde un $50 \%$ declara haber consumido alcohol con una media de 50, error típico de 1 , mediana 50 , una desviación estándar de 1,41 y varianza de la muestra de 2, también declaro un $67 \%$ tener acceso al alcohol con una media de 50 , error típico de 17, mediana 50 , una desviación estándar de 24,04 y varianza de la muestra de 578 , así mismo revelo un 59\% consumir alcohol en lugares públicos(discoteca) con una media de 50, error típico de 9, mediana 50 , una desviación estándar de 12,72 y varianza de la muestra de 162.

En el gráfico 3. De acuerdo a los encuestados, en relación a los factores socio familiares fueron favorables en un 52\% (91 estudiantes) un $48 \%$ (83 estudiantes) de factores desfavorables, con una media aritmética de 50 , un error típico 2, una mediana 50, una desviación estándar 2,82 y varianza de la muestra 8.

En el grafico 4. Del $100 \%$ de adolescentes que consumen alcohol según edad de inicio fue antes de los 15 años alcanzando 73\% (127 estudiantes) y la causa del consumo por primera vez fue por curiosidad alcanzando 94\% (164 estudiantes) sin embargo el consumo actual es por presión de los amigos en el 97\% (169 estudiantes)

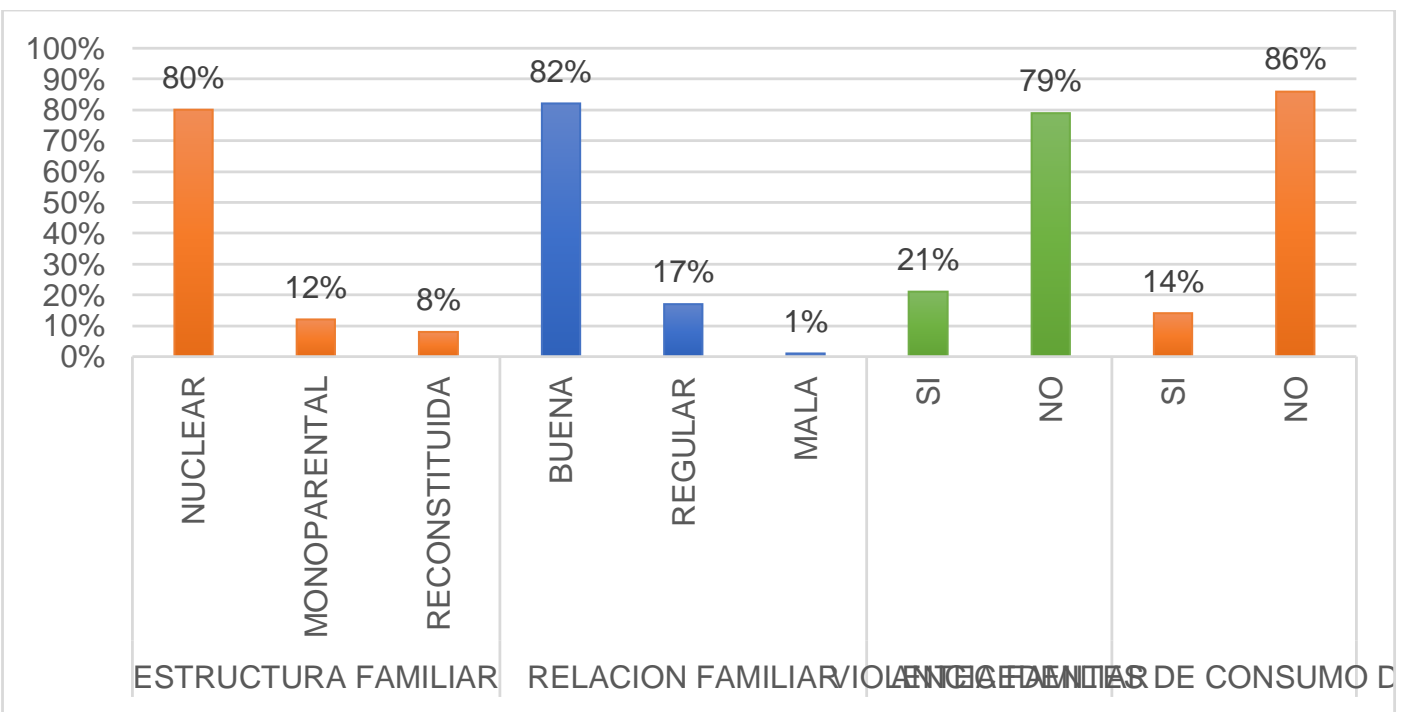

Gráfico 1. Factores familiares de los adolescentes 


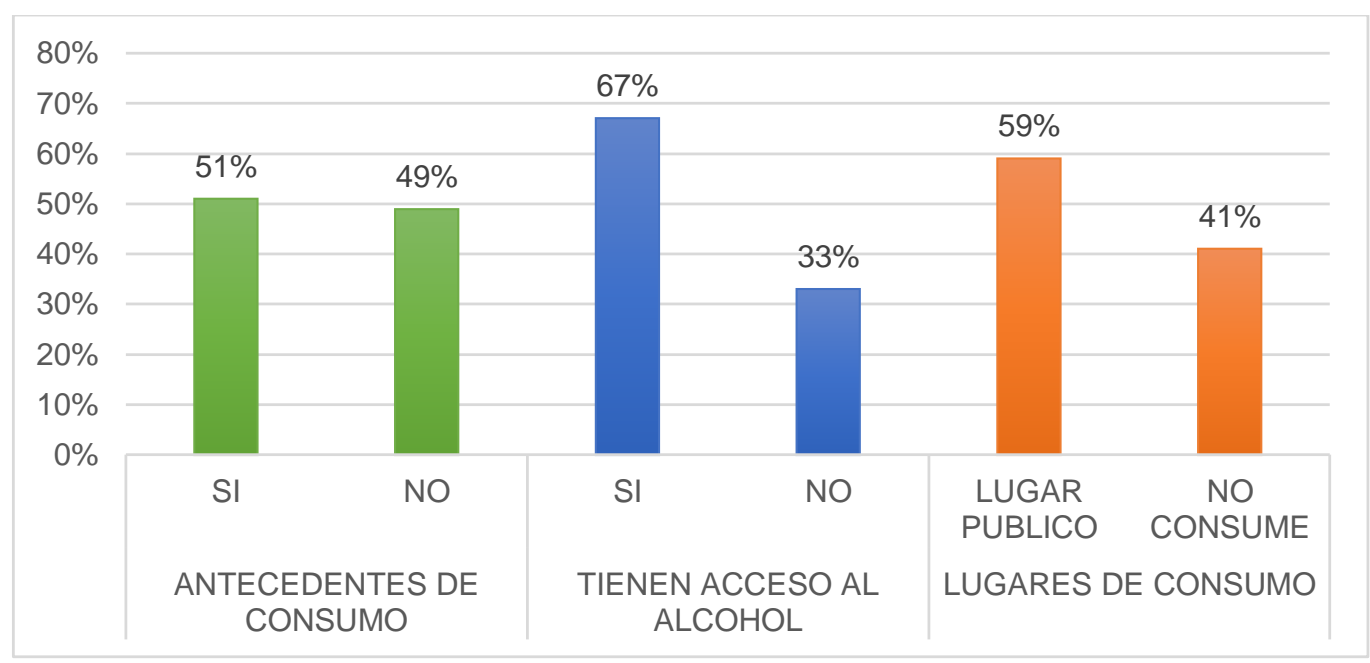

Gráfico 2. Factores interpersonales de los adolescentes

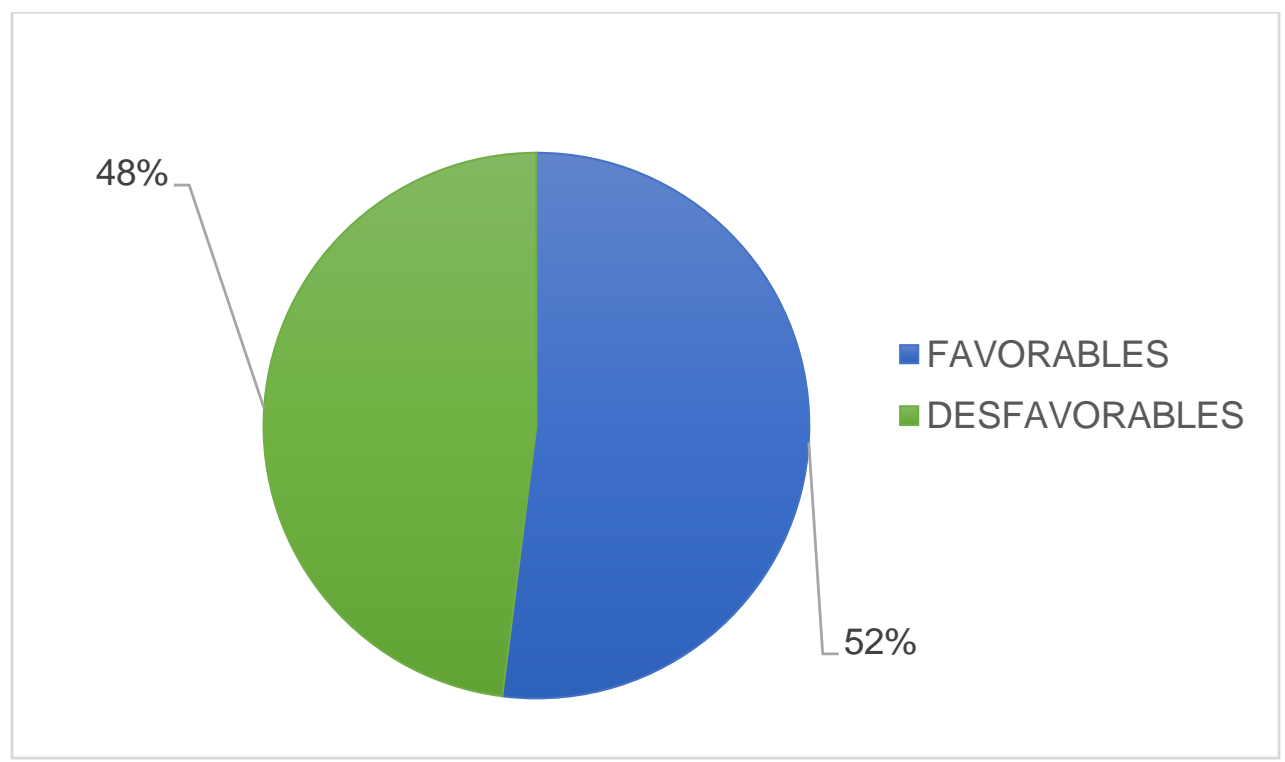

Gráfico 3. Factores socio familiares de los adolescentes

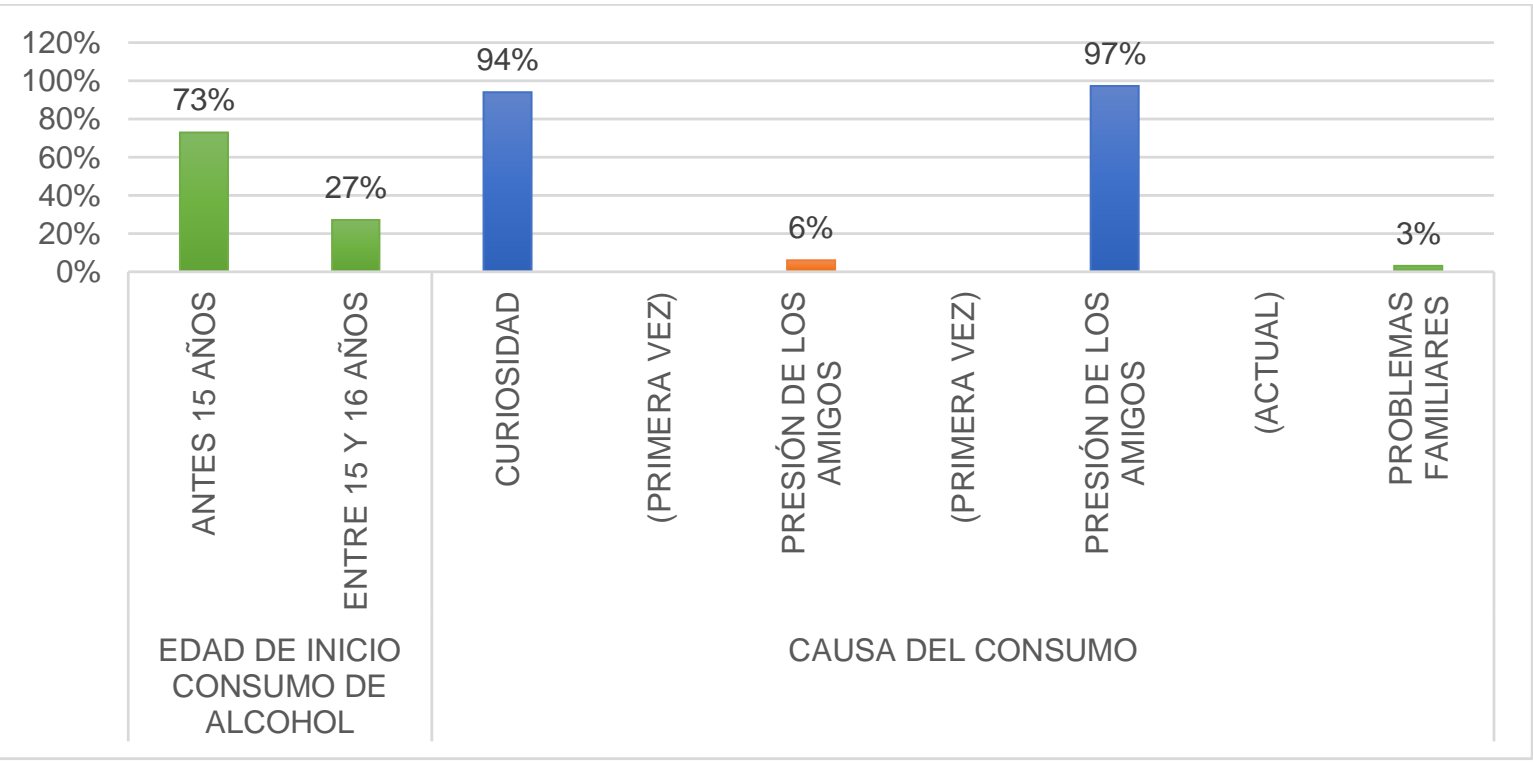

Gráfico 4. Consumo de alcohol según edad de inicio y su causa en los estudiantes 
En el grafico 5. Del 100\% de adolescentes que consumen alcohol según la frecuencia del consumo de alcohol fue de 2 veces en la semana alcanzando $41 \%$ (71 estudiantes), en tanto que la compañía de consumo fueron los amigos de barrio en el $46 \%$ (80 estudiantes).

En el gráfico 6. El consumo de alcohol en los estudiantes según lugar de consumo fue en discotecas alcanzando 38\% (66 estudiantes) siendo el tipo de bebida de mayor consumo la cerveza $51 \%$ (88 estudiantes).

En el grafico 7. Del $100 \%$ de estudiantes que consumen alcohol, existe un 55,7\% (97 estudiantes) que tiene un alto consumo, un $23,7 \%$ (41 estudiantes) tiene un consumo medio y solo el $20,6 \%$ (36 estudiantes) tiene un consumo bajo, lo que demuestra como asciende el consumo de alcohol en los estudiantes.

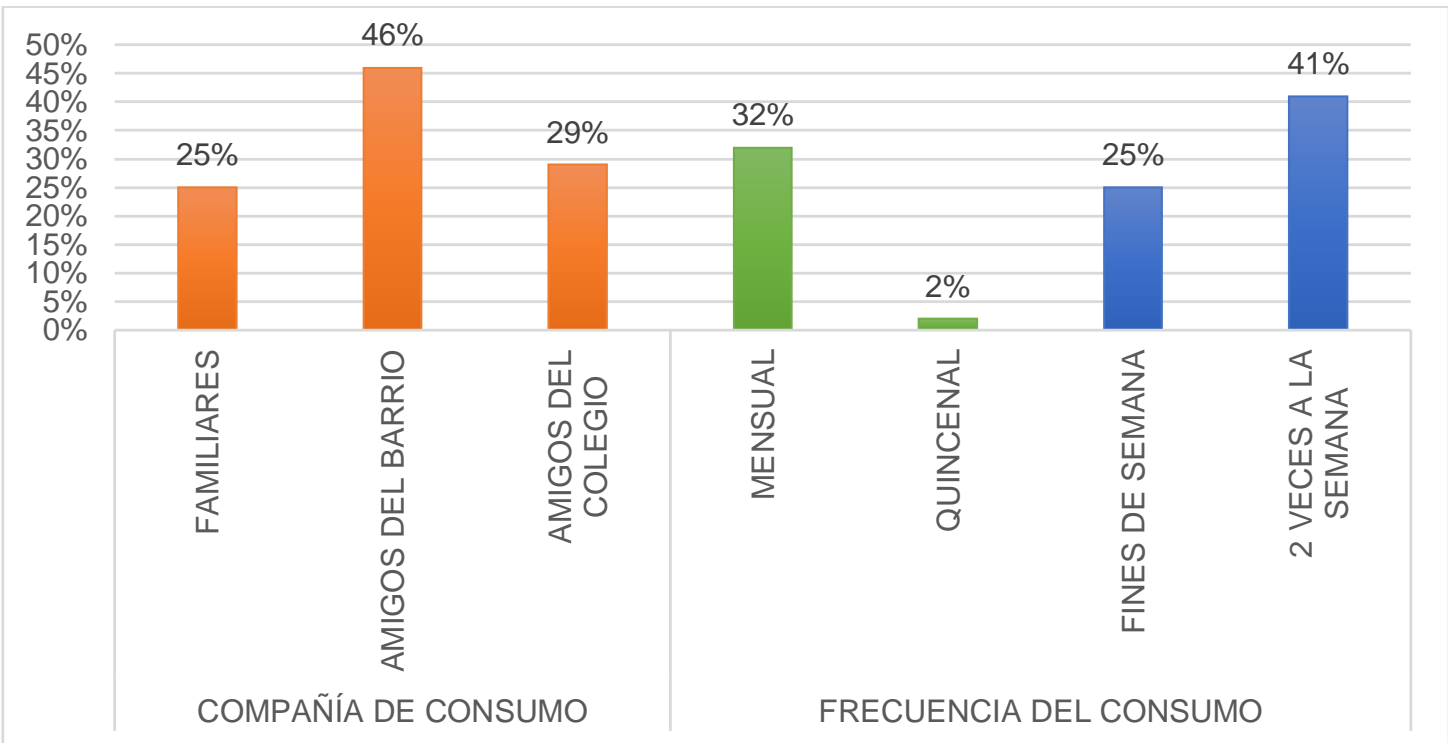

Gráfico 5. Consumo de alcohol según frecuencia y compañía de consumo en los estudiantes

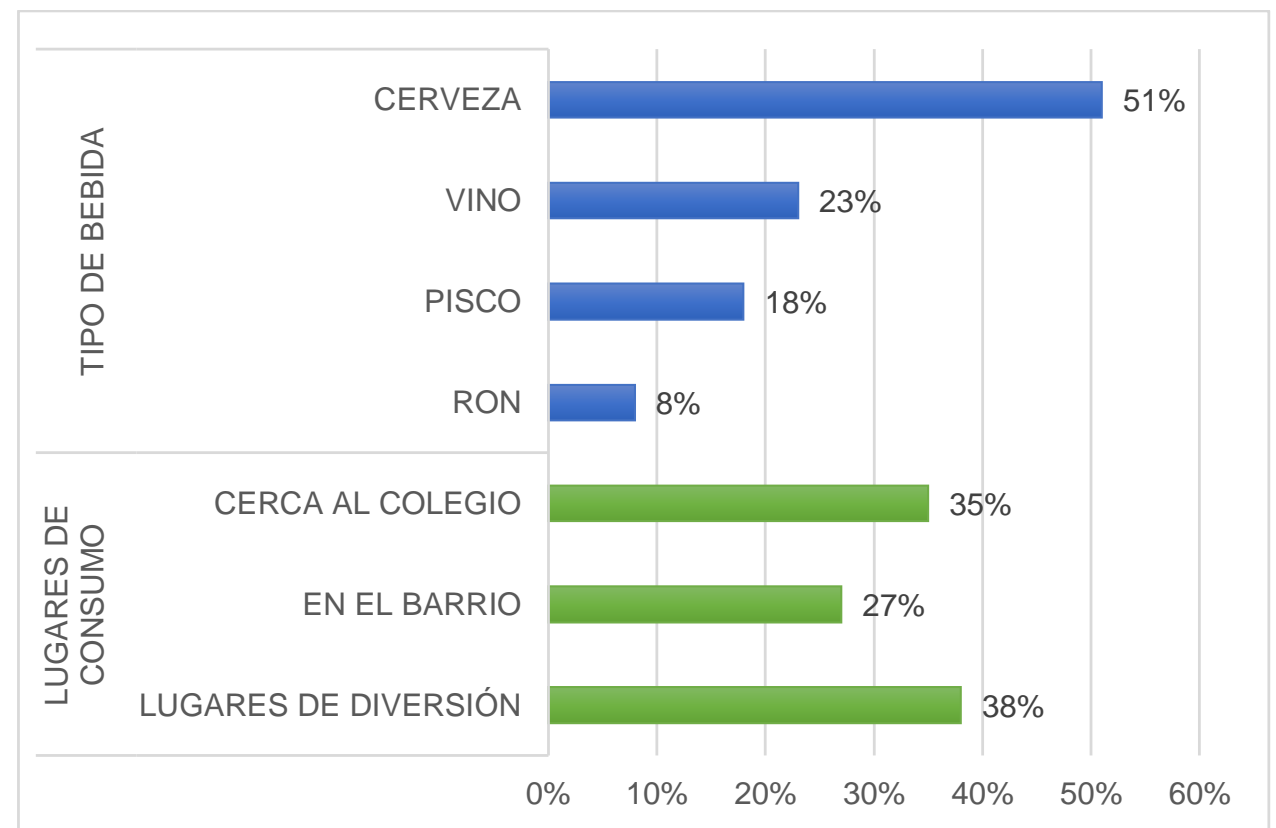

Gráfico 6. Consumo de alcohol según lugares de dispendio y tipo de bebida 


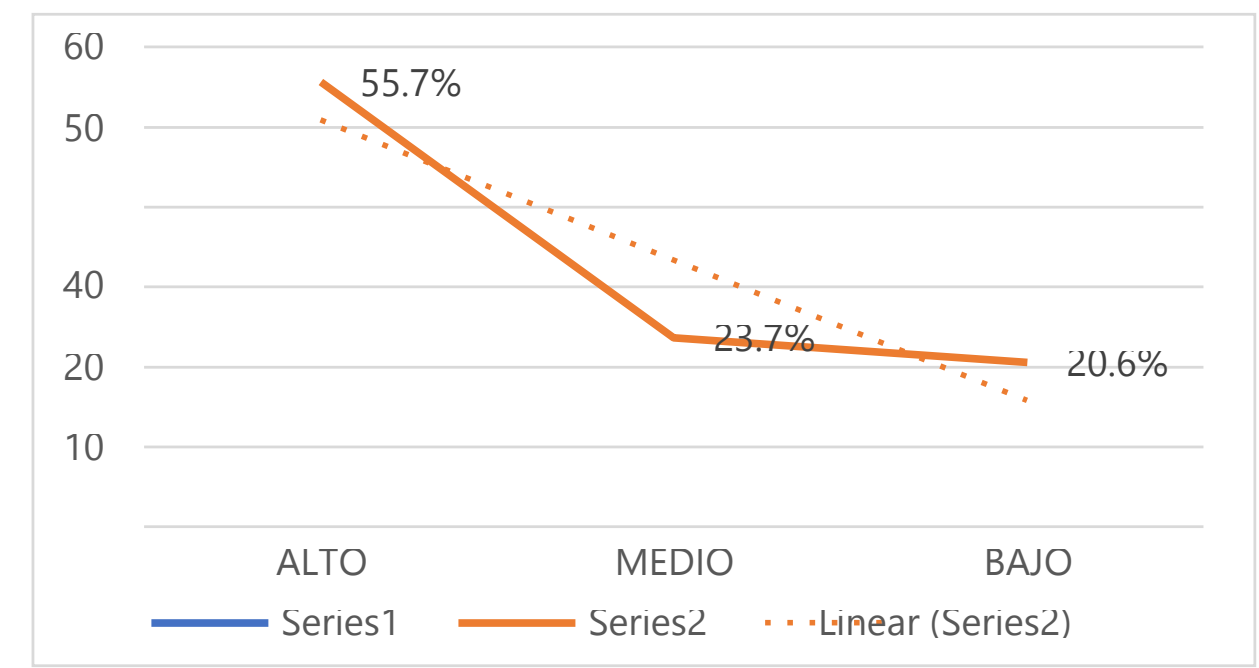

\section{Gráfico 7. Consumo de alcohol en los adolescentes dela institución Educativa Particular "Jesús Maestro" Ica}

\section{DISCUSIÓN}

En la actualidad los estudios sobre consumo de alcohol en los diferentes contextos sean internacional como en Latinoamérica se vienen desarrollando. En el estudio la herramienta utilizada permitió aproximarnos de una manera fáctica en cuanto a los factores socio familiares y su influencia en el consumo de alcohol de los estudiantes evaluados. Datos Generales, (Gráfico1) El promedio de edad oscila entre los 15 a 17 años (56\%) con una desviación estándar del 8,48 , donde los factores familiares fueron desfavorables en $34 \%$, así mismo los factores interpersonales fueron desfavorables alcanzando $60 \%$, destacándose que el $51 \%$ de los estudiantes consume o ha consumido alcohol, un $67 \%$ tienen acceso al alcohol y un $59 \%$ de los estudiantes consume en lugares públicos.(Gráfico 4), teniendo como principal causa de consumo de bebidas alcohólicas la presión de los amigos (97\%) (Gráfico 4), siendo con mayor frecuencia su compañía de consumo los amigos del barrio $46 \%$ (Gráfico 5), su bebida alcohólica preferida es la cerveza $51 \%$ y como lugar preferido de consumo de bebidas alcohólicas la discoteca (38\%).Coincidiendo con Rueda indicando que uno de cada siete estudiantes presentó un patrón de consumo abusivo de alcohol (9), así mismo Camacho et, al. señala que la influencia de los amigos y las expectativas resultaron ser relevantes en su relación con el consumo de alcohol en adolescentes(10), coincidiendo con Cabanillas revelando que la edad de inicio fue de11 a 13 años, y primer año de secundaria (2) Pons detectó que el consumo de alcohol en los adolescentes está significativamente relacionado con el consumo de los otros miembros de su familia, y con la utilización por parte de los padres de estrategias educativas basadas principalmente en la reprobación y en la ausencia de comprensión y afecto. (11). Así mismo Valdez coincide al señalar que el consumo de alcohol se presenta de forma similar entre hombres y mujeres, existiendo diferencias en el consumo toda vez que los hombres revelen más abuso del alcohol, existiendo una tendencia alta por reflejar el incremento del consumo de alcohol. (12)

En correspondencia con Álvarez existen otros factores como haber padecido malos tratos durante la infancia, no sólo de tipo sexual o físico, sino también en forma de negligencia o abuso emocional, hacen que las probabilidades de consumo de sustancias aumenten, como la presencia de ciertos trastornos mentales y convivir con un progenitor que presenta problemas de consumo de alcohol hacen que también se aumenten visiblemente los riesgos de 
consumo de bebidas alcohólicas durante la adolescencia (13) en correlación con De los Àngels, quien describe otro factor importante a considerar es lo que se refiere al aumento de conductas de riesgo y psicopatología en la adolescencia es un signo de alarma al cual debemos responder con acciones concretas y viables, expone que el alcohol ha tenido un gran repunte en el consumo dentro de los adolescentes sobre todo en la primera etapa de la adolescencia que es ratificado en el estudio(14).

\section{CONCLUSIONES}

1. De la contrastación de hipótesis el valor obtenido supero el valor crítico, rechazando la hipótesis nula aceptando que los factores socio familiares influyen significativamente en el consumo de alcohol en los estudiantes.

2. Los factores socio familiares (Estructura familiar, relación familiar, violencia familiar, antecedentes de consumo familiar) fueron favorables en un $52 \%$ existiendo solo el $48 \%$ de factores desfavorables.

3. Los factores interpersonales (Antecedentes de consumo de amigos acceso al alcohol, lugares de consumo) fueron desfavorables alcanzando un $60 \%$ (105).

4. Del $100 \%$ de adolescentes del colegio particular "Jesús Maestro" existe un 55.7\% tienen un alto consumo de alcohol, Observándose que el alcohol ha tenido un gran repunte en el consumo dentro de los adolescentes sobre todo en la primera etapa de la adolescencia.

\section{RECOMENDACIONES}

1. Impulsar la participación activa de los padres de familia, toda vez que son agentes activos de cambio, porque son los que están en mayor contacto con los estudiantes y pueden llegar a ser figuras importantes de autoridad y de confianza.

2. Instalar áreas de salud en los colegios exclusivas para la atención directa a los estudiantes, donde puedan ser atendidos de manera psicológica y fisiológica también a sus familiares.

3. Los resultados del estudio también nos muestran muy claramente que se debe ejercer presión social a cerca de la Ley N²8681, encargada de regular la comercialización, consumo y publicidad de bebidas alcohólicas a fin de proteger a los menores de edad., no permitiéndoles el ingreso a lugares públicos como discotecas $\mathrm{u}$ otros donde se expenda bebidas alcohólicas.

4. La caracterización de los estudiantes referidos al alto consumo de alcohol, en el instituto privado Jesús Maestro de Ica permitirá la necesidad de una mayor difusión de programas por el Estado, profesores, familia y personal de salud, en donde el alcoholismo sea tratado desde los diferentes puntos que afectan al adolescente como Biológico, Psicológico y emocional, además la ayuda oportuna de profesionales de la salud en distintas especialidades para que los adolescentes puedan tomar conciencia de su situación y del daño que hace a su presente y futuro

\section{CORRESPONDENCIA:}

Dr. Héctor William Carlos Cruces.

Correo electrónico:

Hector.carlos@unica.edu.pe

Celular: 921408176

\section{REFERENCIAS BIBLIOGRÀFICAS}

1. Organización Mundial de la Salud. (OMS) Informe de situación consumo de alcohol 21 de setiembre 2018

2. Cabanillas W. Consumo de alcohol y género en la población adolescente escolarizada del Perú: evolución y retos de intervención Rev Perú Med Exp Salud Publica. 2020; 37(1):148-54

3. Comisión Nacional para el Desarrollo y Vida sin Drogas. Estudio nacional sobre prevención y consumo de drogas en 
estudiantes de secundaria 2017. Lima: DEVIDA; 2019

4. Biolatto L. Estrategias con adolescentes para prevenir el consumo problemático de alcohol: modalidades y ámbitos de aplicación. Hacia Promoc. Salud. 2018; 23 (2): 48-66. DOI: 10.17151/hpsal.2018.23.2.4

5. Tegoma V., Cortaza I. Prevalencia del consumo de alcohol en adolescentes de secundaria de Coatzacoalcos, Veracruz, Mexico.Rev. Enferm. univ.2016;13(4):

6. Flores J. Factores de riesgo asociados al consumo de bebidas alcohólicas en los adolescentes de dos instituciones educativas una pública y una prada; 2016

7. Organización de las Naciones Unidas para la Educación, la Ciencia y la Cultura (UNESCO) ¿Cómo el sector de la educación debe hacer frente al consumo de alcohol, tabaco y drogas? 2018

8. Ñaupas H, Mejía E, Novoa E, Villagómez

A. Metodología de la investigación cuantitativa-cualitativa y redacción de la tesis $5^{\mathrm{a}}$ ed. Colombia; Ed. Ediciones de la U.; 2018

9. Rueda G. et. al. Prevalencia del abuso de alcohol en niños y adolescentes escolarizados de Bucaramanga, y sus factores asociados. Investigación institucional Colombia; 2012.
10. Camacho L, Ibáñez MI, Mezquita L, Viruela A, Gallego S, Moya J. et al. Influencia de los amigos y las expectativas en el consumo de alcohol en adolescentes. [Producción intelectual] España; 2012.

11. Pons D. Javier El modelado familiar y el papel educativo de los padres en la etiología del consumo de alcohol en los adolescentes.2014

12. Valdez J. El consumo de alcohol en estudiantes del Distrito Federal y su relación con la autoestima y la percepción de riesgo.2005

13. Álvarez A. María J. Propuesta de un modelo predictivo del consumo temprano de alcohol en adolescentes.2015

14. De los Àngels $P$. María Factores de Riesgo y Factores de Protección en la Adolescencia: Análisis de Contenido a través de Grupos de Discusión.2011

Recibido: 05 /03/ 2021

Aprobado para publicación: 19/08/2021 